\title{
Refractive outcomes of cataract surgery in primary congenital glaucoma
}

\author{
Sudarshan Khokhar ${ }^{1} \cdot$ Dev Yadav $^{1} \cdot$ Shikha Gupta $\mathbb{B}^{1} \cdot$ Ramanjit Sihota $^{1} \cdot$ Abadh Kishore Chaurasia $^{1} \cdot$ \\ Amisha Gupta ${ }^{1}$ V Viney Gupta (1) ${ }^{1}$
}

Received: 19 September 2017 / Revised: 20 July 2018 / Accepted: 5 August 2018 / Published online: 31 October 2018

(c) The Royal College of Ophthalmologists 2018

\begin{abstract}
Aim To evaluate refractive outcomes of cataract surgery with intraocular lens (IOL) implantation in operated eyes of primary congenital glaucoma (PCG).

Design A retrospective case-control study.

Methods Patients of PCG who developed cataract following trabeculectomy with trabeculotomy were recruited. Preoperative biometry was recorded and refractive outcomes of the patients in terms of spherical equivalent (SE) and prediction error were noted at 3 and at 12 months following surgery. The refractive outcomes were compared with non-glaucomatous eyes of children in similar age group who underwent lens aspiration with IOL implantation (controls).

Results The median age of the children with PCG $(n=31)$ at the time of cataract surgery was 60 months, similar to controls $(n=29) ; 48$ months $(p=0.3)$. The SE in PCG eyes at 12 months was comparable to controls $(p=0.18)$. The prediction error (postoperative SE - predicted SE) at 3 months $(p=0.018)$ and at 12 months $(p=0.03)$ among PCG eyes was higher and more myopic compared with controls. The range of prediction error at 12 months in PCG eyes was -8.6 to $+5.8 \mathrm{D}$ (median $-2.0 \mathrm{D}$ ), whereas in controls it was -4.2 to $+6.3 \mathrm{D}$ (median $+0.5 \mathrm{D}$ ). For each mmHg intraocular pressure (IOP) increase there was $0.42 \mathrm{~mm}$ increase in axial length among PCG eyes and a $0.24 \mathrm{~mm}$ increase among controls $(p<0.001)$. Conclusions After IOL implantation there was a greater prediction error and a greater myopic shift among PCG eyes. Eyes of children with PCG are more prone to refractive surprises as their axial length changes are more sensitive to IOP fluctuation.
\end{abstract}

\section{Introduction}

Primary congenital glaucoma (PCG) is uncommon with prevalence varying in different populations [1-3]. Different surgical options are used to treat PCG depending on the severity and choice of the surgeon $[4,5]$. Glaucoma surgery increases the incidence of cataract formation. Among adults, glaucoma surgery is associated with greater incidence of cataractogenesis compared with medical treatment $[6,7]$.

Electronic supplementary material The online version of this article (https://doi.org/10.1038/s41433-018-0253-6) contains supplementary material, which is available to authorised users.

Viney Gupta

gupta_v20032000@yahoo.com

1 Dr. Rajendra Prasad Centre for Ophthalmic Sciences, All India Institute of Medical Sciences, New Delhi, India
However, there are no studies that have evaluated the incidence of cataract formation after surgical treatment of PCG.

Once cataract develops in a child operated for PCG, cataract surgery has its own set of challenges, different from eyes of children without glaucoma. One of the challenges is obtaining desirable refractive outcomes post cataract surgery with intraocular lens (IOL) implantation in these eyes. To the best of our knowledge, no studies have reported refractive outcomes in PCG eyes after IOL implantation. We thus aimed to evaluate the refractive outcomes of cataract surgery and IOL implantation in PCG eyes that had developed cataract after a combined trabeculotomy and trabeculectomy.

\section{Patients and methods}

This was a retrospective case-control study. The study was conducted after approval from our Institutional Ethics 
Committee. The study conformed to the tenets of the Declaration of Helsinki. A total of 31 eyes of PCG with controlled intraocular pressure (IOP; $<21 \mathrm{mmHg}$ with or without topical medication as measured under general anaesthesia using Perkins tonometer) after filtering surgery (combined trabeculotomy with trabeculectomy) who underwent a lens aspiration with posterior chamber IOL implantation were included in our study as cases and 29 eyes of congenital cataract who had undergone lens aspiration with IOL implantation during the same period were included as controls. All surgeries performed by a single surgeon (SK), from Jan 2010 to Jan 2015, were reviewed. The predicted (calculated) and actual refractive outcomes of the patients in terms of spherical equivalent (SE) and lens power prediction error were noted at 3 and at 12 months following surgery. The refractive outcomes were compared with non-glaucomatous eyes of children in similar age group who underwent lens aspiration with IOL implantation (controls).

Inclusion criteria (PCG eyes)

- Patients with successful trabeculectomy with trabeculotomy, in the past, who underwent lens aspiration with IOL implantation for a visually significant cataract. A visually significant cataract was considered when the lens opacity covered the entire undilated portion of the pupil.

- A minimum duration of 3 months between filtering surgery and cataract surgery.

- Patients aged 10 yrs or less.

\section{Exclusion criteria (PCG eyes)}

- Failed filters (those in whom IOP was $\geq 21 \mathrm{mmHg}$ despite topical anti glaucoma therapy or those with an increase in their axial length, corneal diameter or worsening of cup disc ratio over past 6 months).

- Eyes with media opacity that precluded refraction.

- Eyes operated for secondary causes of congenital glaucoma.

Inclusion criteria (controls)

- Patients $<10$ years of age who underwent lens aspiration with IOL implantation for a visually significant congenital cataract.

Exclusion criteria (controls)

- Traumatic cataract.

- Complicated cataract.

- Cataracts associated with congenital rubella, retinopathy of prematurity (ROP) or primary hyperplastic posterior vitreous (PHPV).
Preoperative/intra-operative ocular biometry was recorded. The Sanders-Retzlaff-Kraff (SRK) II formula was used for IOL power calculation and proposed IOL power reduction was based on the surgeon's experience (Suppl Table).

The patients underwent lens aspiration and implantation of a foldable hydrophobic acrylic multi-piece IOL (Acrysof MA60AC, Alcon Laboratories, USA), into the capsular bag with optic capture through the posterior capsulorrhexis followed by standard postoperative care. Posterior capsulorrhexis was performed in all eyes and was combined with a limited anterior vitrectomy in children $<8$ years of age.

One eye of each child was analysed. If the child had been operated in both eyes for cataract surgery, the right eye was selected for the study. Preoperative records were analysed to look for the position of Haab's striae and the refractive error before the cataract surgery.

Data collected included age at the time of cataract surgery, preoperative/intra-operative axial length, keratometry (using a portable keratometer) and IOP. During follow-up examination under anaesthesia (EUA) at 3 months, and 12 months following parameters were assessed: refraction, Perkins' applanation tonometry, an A scan Ultrasound measurement of axial length, keratometry and indirect ophthalmoscopy for fundus examination. Children who were found to have increased IOP postoperatively underwent EUA more frequently to monitor their IOP and Biometric parameters.

Refraction was performed using hand held auto refractor (Nikon Corporation, Tokyo Japan) and rechecked with manual retinoscopy at each visit. The SE of the refractive error was taken by adding half of the cylinder to the sphere. Prediction error was calculated as the difference of the predicted from the actual SE (postoperative/actual SE predicted/calculated SE). We used SPSS version 20 (IBM, Somers, New York, USA) for statistical analysis.

\section{Results}

The demographic and preoperative clinical details of the PCG and control eyes are given in Table 1. There was a male preponderance among the PCG group. The median age of PCG patients was greater than controls by 12 months, though the difference was not significant $(p=$ $0.3)$. The PCG eyes also had greater axial length (AL), flatter corneal curvature and greater corneal diameters compared with controls. Before the cataract surgery the refractive error (SE) was the following: 14 eyes had a myopia $>3 \mathrm{D}, 10$ eyes had myopia between 1 and $3 \mathrm{D}$ and 7 eyes were hyperopic upto $3 \mathrm{D}$.

In PCG eyes, the average AL increased from $25 \pm 2.36$ $\mathrm{mm}$ to $26.4 \pm 2.1 \mathrm{~mm}(p<0.001)$ at 12 months while there 
Table 1 Demographic, preoperative and postoperative clinical characteristics of PCG and control eyes

\begin{tabular}{|c|c|c|c|}
\hline & PCG $(n=31)$ & $\operatorname{Controls}(n=29)$ & $P$ value \\
\hline \multicolumn{4}{|l|}{$\begin{array}{l}\text { Demographic and preoperative } \\
\text { characteristics }\end{array}$} \\
\hline Gender (M:F) & $27: 4$ & $12: 17$ & 0.001 \\
\hline Mean age at cataract surgery \pm SD (months) & $59.9 \pm 43.6$ & $48.1 \pm 34.2$ & \\
\hline $\begin{array}{l}\text { Median age at cataract surgery in months } \\
\text { (range) }\end{array}$ & $60(10-168)$ & $48(8-112)$ & $0.3 *$ \\
\hline Avg corneal diameter $(\mathrm{mm})$ & $13.51 \pm 1.14$ & $11.35 \pm 0.73$ & 0.036 \\
\hline Avg axial length (mm) & $25 \pm 2.36$ & $21.85 \pm 1.58$ & 0.027 \\
\hline Avg keratometry (D) & $40.4 \pm 3.3$ & $43.95 \pm 2.14$ & 0.029 \\
\hline Avg intraocular pressure $(\mathrm{mmHg})$ & $14.54 \pm 4.53$ & $10.75 \pm 1.55$ & 0.01 \\
\hline Avg cup disc ratio & $0.7 \pm 0.23$ & $0.3 \pm 0.11$ & 0.005 \\
\hline \multicolumn{4}{|l|}{ Postoperative characteristics } \\
\hline $\begin{array}{l}\text { Avg intraocular pressure at } 3 \text { months } \\
(\mathrm{mmHg})\end{array}$ & $15.5 \pm 8.3$ & $12.6 \pm 2.5$ & 0.01 \\
\hline $\begin{array}{l}\text { Spherical equivalent ( } 3 \text { months); median } \\
\text { (range) }\end{array}$ & $\begin{array}{l}+1.5 \mathrm{D}(+3 \text { to }+0.5 \\
\mathrm{D})\end{array}$ & $3.1 \mathrm{D}(+8.5-0 \mathrm{D})$ & $0.055^{*}$ \\
\hline $\begin{array}{l}\text { Spherical equivalent (12 months); median } \\
\text { (range) }\end{array}$ & $\begin{array}{l}+0.25 \mathrm{D}(+1.5 \text { to }- \\
2.5 \mathrm{D})\end{array}$ & $\begin{array}{l}+1.2 \mathrm{D}(+6.5 \text { to }-0.5 \\
\text { D) }\end{array}$ & $0.18^{*}$ \\
\hline Prediction error ( 3 months); median (range) & $+0.2 \mathrm{D}(+4$ to $-6 \mathrm{D})$ & $\begin{array}{l}+2 \mathrm{D}(+6 \mathrm{TO}-2.5 \\
\mathrm{D})\end{array}$ & $0.018^{*}$ \\
\hline $\begin{array}{l}\text { Prediction error (12 months); median } \\
\text { (range) }\end{array}$ & $\begin{array}{l}-2 \mathrm{D}(-8.6 \text { to }+5.8 \\
\mathrm{D})\end{array}$ & $\begin{array}{l}+0.5 \mathrm{D}(-4.2 \text { to }+6.3 \\
\text { D) }\end{array}$ & $0.03 *$ \\
\hline
\end{tabular}

*Using Mann-Whitney test

was no significant change in the average keratometry; from $40.4 \pm 3.3 \mathrm{D}$ to $40.2 \pm 3.1 \mathrm{D}(p=0.1)$. Even in the control eyes there was an increase in AL over 12 months; $21.85 \pm$ $1.58 \mathrm{~mm}$ to $22 \pm 1.4 \mathrm{~mm}(p=0.007)$ but no change in keratometry was noted; $43.95 \pm 2.14 \mathrm{D}$ to $43.8 \pm 2.3 \mathrm{D}(p=$ 0.12). All PCG eyes were noted to have Haab's striae, The position of the Haab's striae was central (involving the pupil) in 6 of the 31 eyes, whereas in the other eyes they were away from the central zone of the pupil.

To assess the effect of age at surgery on the refractive error, the PCG and control eyes were divided into those $<2$ years, 2-8 years and those above 8 years. The SE at 3 and 12 months among PCG eyes and controls in different age groups is shown in Fig. 1a, b. The median SE in PCG eyes at 12 months was $+0.25 \mathrm{D}$ comparable to controls; $+1.2 \mathrm{D}$ (Mann-Whitney $U=286, p=0.18$ ). The average under correction of the implanted IOL among controls was +6.2 $\pm 1.8 \mathrm{D},+2.8 \pm 2 \mathrm{D}$ and $+1 \pm 0.5 \mathrm{D}$ for $<2$ years age, $2-8$ years age and $>8$ years age group, respectively. The average under correction of the implanted IOL among PCG eyes was $+2.1 \pm 2 \mathrm{D},+1.7 \pm 1.2 \mathrm{D}$ and $+1.1 \pm 1 \mathrm{D}$ for $<2$ years age, $2-8$ years age and $>8$ years age group, respectively. The surgeon under correction for PCG eyes was expectedly lower as these eyes have larger Axial Lengths making the IOL power lower and the under correction lower (Suppl Table) in comparison with control eyes that have proportionately smaller (albeit normal) axial lengths. a

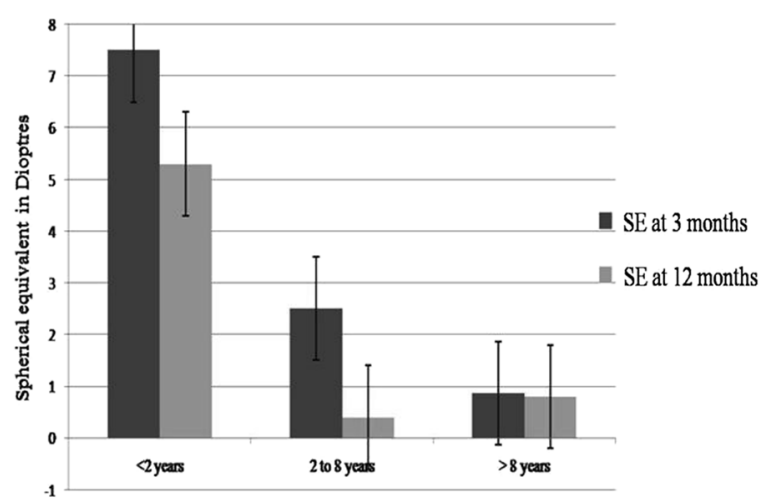

b

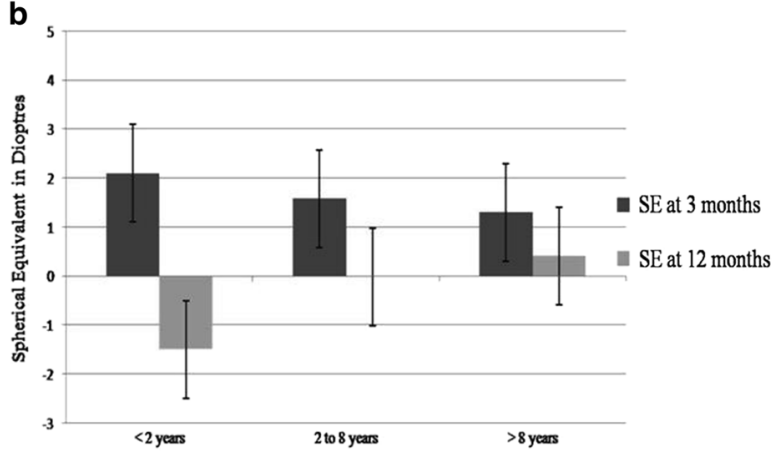

Fig. 1 a Spherical equivalent (SE) at 3 and 12 months in different age groups among controls. Figure $1 \mathrm{~b}$ among PCG eyes 


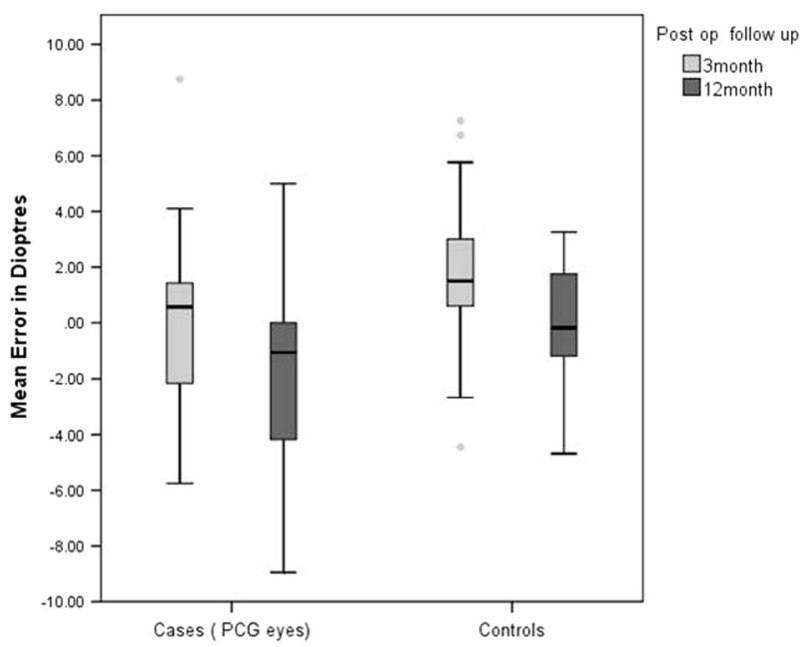

Fig. 2 Mean prediction error (postoperative SE minus the predicted $\mathrm{SE})$ over time, among PCG eyes and controls

The prediction error (postoperative SE - predicted SE) at 3 months $(U=290, p=0.018)$ and at 12 months $(U=243$, $p=0.03$ ) among PCG eyes had a greater disparity and was more myopic compared with controls (Fig. 2). At 12 months after surgery, the range of prediction error (postoperative $\mathrm{SE}-$ predicted SE) in control group was $-4.6 \mathrm{D}$ to $+3.3 \mathrm{D}$ (median: $+0.5 \mathrm{D}$ ) and in PCG eyes was $-8.6 \mathrm{D}$ to $+5 \mathrm{D}$ (median $-2.0 \mathrm{D})$.

The absolute prediction error (irrespective of myopia/ hyperopia) was categorised as $<1 \mathrm{D}, 1-3 \mathrm{D}$ and $>3 \mathrm{D}$. We aimed to look for the proportion of eyes having prediction errors in these categories in the two groups. At 12 months postoperatively, the proportion of eyes showing refractive errors $>3 \mathrm{D}$ were more in PCG eyes than controls $(p=0.01)$ as shown in Fig. 3. The proportion of eyes showing a refractive surprise $>3 \mathrm{D}$ was not different between the group that had central Haab's striae $(n=6)$ compared with those that were away $(n=25)$ from the pupillary zone $(p=0.56)$. To look for whether the preoperative refractive error was associated with the postoperative outcomes, we divided those with a preoperative refractive error $>3 \mathrm{D}(n=14)$ and those with less $<3 \mathrm{D}$ $(n=17)$. The ones that showed a refractive surprise were not different from those that did in terms of the preoperative refractive error $(p=0.9)$.

The refractive surprise for all eyes (cases and controls) was plotted as a function of IOP (Fig. 4). The preoperative IOP was plotted against postoperative IOP. The circles represented the refractive error (small and large) of the individual cases. We found that the eyes with greater refractive surprise (larger circles, $\geq 3 \mathrm{D}$ ) were more frequently seen when IOP was increased postoperatively (lying above the intersecting/oblique line). The postoperative rise in IOP was managed medically.

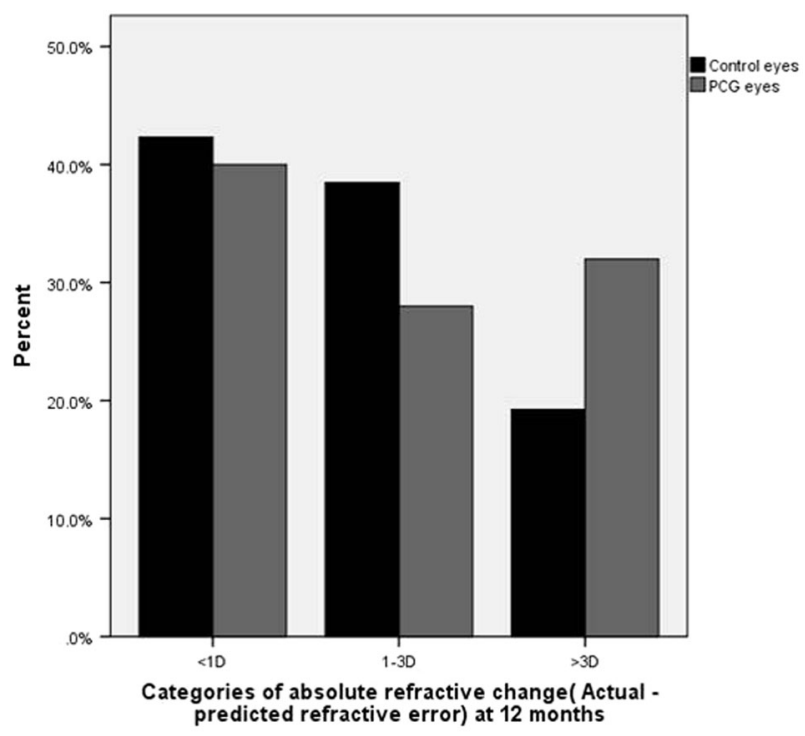

Fig. 3 Categories of absolute prediction error (postoperative SE minus the predicted SE) at 12 months among PCG eyes and controls

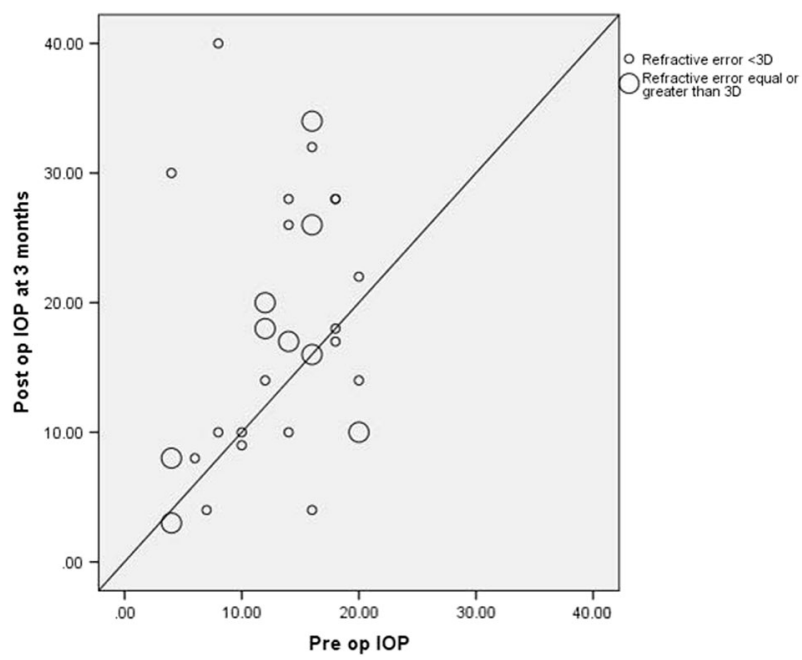

Fig. 4 Refractive error as a function of IOP for all eyes. The larger circles represent refractive errors $\geq 3 \mathrm{D}$. Most larger circles are seen to lie above the intersecting line indicating that refractive error is higher if IOP rises post cataract surgery

In our study, controls had $0.34 \mathrm{~mm} /$ year increment in axial length in age group below 2 years, $0.26 \mathrm{~mm} /$ year in age group 2-8 years and minimal or no growth in age group above 8 years. Among PCG eyes increase in AL below 2 years was $1.48 \mathrm{~mm}$ per year, $1.27 \mathrm{~mm}$ per year in age group $2-8$ years and $0.82 \mathrm{~mm}$ per year in those $>8$ years old. The change in $\mathrm{AL}$ at 12 months from the preoperative values was calculated and divided by the change in IOP during the same period for both controls and PCG eyes. We found that, for each $\mathrm{mmHg}$ of IOP increase in PCG eyes there was a $0.42 \mathrm{~mm}$ increase in axial length and a $0.24 \mathrm{~mm}$ increase among controls overall $(p<0.001)$. 


\section{Discussion}

The eyes of children grow throughout childhood and have the greatest changes within the first 2 years of life. Recognising the nonlinear growth patterns of children's eyes, cataract surgery with choosing the best lens implant power for children is fraught with difficulty. This is especially so in eyes with PCG having poor pressure control, resulting in changes in axial length and changes in refraction. With the above stated, when children's eyes with glaucoma require cataract surgery and lens implant, selection of the optimal lens power may be very challenging. To address these issues, we studied a group of children under 10 years of age who had had glaucoma surgery and developed cataracts and compared them with a control group of children with cataracts but without glaucoma. Our goal was to investigate the changes in the refractive error by analysing the 'prediction error'. The prediction error was the difference in the calculated IOL power as determined by the SRK II formula and compare results with the actual refraction achieved at 3 months and 12 months following surgery. We categorized three age groups, under 2 years, $2-$ 8 years and $>8$ years, for comparison between the cases and controls. In this study, we also tried to clarify issues of growth of the eyes and changes in refraction encountered in eyes with glaucoma and to offer some guidelines to assist in choosing the optimal IOL power in children who have PCG and cataracts in the first decade of life.

In PCG eyes, after surgery for IOP control, the AL stabilises, if the IOP is controlled or may even reduce, which results in variation in refraction with time $[8,9]$. This has also been shown among adults after filtering surgery [1012]. However, after cataract surgery, as we have shown, the AL can grow and compound the changing refractive errors in these children.

In our study, the prediction error was significantly more among PCG eyes that also had greater variability in their refractive error compared with the control eyes. The proportion of eyes showing a prediction error of $3 \mathrm{D}$ or more at 12 months was higher among the PCG eyes in comparison with controls. There could be many factors contributing to this including, inaccurate AL measurement on ultrasound, lesser under correction, greater astigmatism in these eyes because of irregularity of cornea due to Haab's striae, and a rapid eye growth. The IOL power to be implanted in a child needs careful consideration. It is difficult to decide about residual refractive error to be left after cataract surgery in children because the blur induced postoperatively may affect the growth of the eye which in turn may lead to myopic shifts in pseudophakic eyes of children. Some surgeons are recently targeting emmetropia after surgery at all ages beyond infancy to counteract amblyopia, but the risk of large myopic shift later in life remains [13].
However, most surgeons aim for hypermetropia up to $8-10$ years of age, as large myopic shifts are known especially in children $<2$ years of age $[14,15]$. Different studies provide different guidelines for IOL power reduction depending upon the age of the child and axial length. To counteract the residual refractive error in children piggyback implantable collamer lenses have recently been proposed [16]. Enyedi et al. [17] provided an appropriate algorithm for under correction depending on the child's age. In our study, we modified Enyedi's under correction algorithm, based on the surgeons (SK) experience and used the same for deciding the IOL power in PCG eyes and those with congenital cataract. The under correction protocol we used, like that described by Enyedi, was based on the age of the child but differed in that we provided an under correction proportionate to the IOL power calculated and a gradation of under correction in those $<2$ years where the eye growth in maximally expected unlike Enyedi's under correction that changed only by $1 \mathrm{D}$ from 1 to 2 years of age.

We also found greater myopic shift among PCG eyes. This could be owing to many reasons including an increase in axial length seen among the PCG eyes. Axial length measurement in PCG eyes is important because on one hand it explains the amount of myopic shift, on the other it subserves to monitor the progression of glaucoma in children. In our study, the axial length grew significantly more over the study duration of 12 months in PCG eyes compared with controls. This was especially so in PCG children $<8$ years of age. We believe that apart from loss of IOP control this could be owing to greater visual deprivation in PCG eyes leading to a greater increase in axial length [1821]. It is also known that the contralateral eye tends to increase in $\mathrm{AL}$ if the other eye has greater $\mathrm{AL}$, which could also be a factor in PCG eyes [22]. The eye growth among PCG eyes in our study after IOL implantation was higher than reported by Gordon et al. [23] among healthy children. Although most studies have shown that axial length change occurs by $8-10$ years, it has been noticed even up to 15 years of age $[14,15]$.

The axial length in PCG eyes was found to increase more than controls in our study despite the fact that the PCG children were on an average older than controls. We found in this study that the axial length of PCG eyes was more sensitive to changes in IOP, that changed significantly more than control eyes, a factor that needs consideration while calculating IOL power. Eyes with higher postoperative IOP showed greater refractive surprise. It is known that increase in IOP can lead to increase in axial length and vice versa [24]. This may be more pronounced in myopic eyes with low scleral rigidity. It is known that highly myopic eyes are more affected by changes in IOP [25].

We used SRK II formula for IOL power calculation in both PCG eyes and congenital cataract eyes. No formula 
has been proven to be perfect predictor of exact IOL power in paediatric age group, but different studies have shown that Hoffer Q formula is more accurate for short eyes $(<22$ $\mathrm{mm})$ and Holladay2 formula is considered optimal for long eyes (>26 mm) [26-29]. Both these formulas need anterior chamber depth (ACD) values for IOL power calculation. As we did not have intra-operative ACD measurement, we used the SRK II regression formula, which has also been shown to be reasonably accurate for eyes with longer axial length $[30,17]$.

One of the problems of IOL implantation in PCG eyes with large axial lengths and a stretched capsular bag is IOL decentration [31]. Significant IOL decentration was not noted in any of the eyes in our series, as we performed an optic capture with a multi-piece IOL. Other complications known to occur in PCG eyes are, rise in IOP that was seen in a few and managed with topical glaucoma therapy. Retinal detachment is another complication, which occurs with greater frequency among PCG eyes especially after cataract surgery [32] though it was not seen in our study eyes during the 12-month evaluation.

A major limitation of the study is its retrospective nature and the fact that many ophthalmologists were involved in examining these children over time. However, all IOL implantations were performed by the same surgeon using the same IOL correction based on his experience. Another limitation of the study was our inability to measure the anterior chamber depth intra operatively, which could be important, as it is known that eyes with PCG have a deeper anterior chamber. This can alter the effective lens position and the final refractive outcome in these eyes.

In conclusion this study shows that the refractive error after IOL implantation in PCG eyes is more prone to change toward myopia and hence greater preoperative under correction may be desirable in these eyes. Long-term prospective studies are needed to evaluate changes in refractive outcomes with time, as also to evaluate the predictors for refractive outcomes in PCG eyes.

\section{Summary}

\section{What was known before}

- Studies among adults show that there is a higher incidence of cataract surgery among eyes that have undergone filtering surgery.

- The refractive outcomes of such eyes after IOL implantation are different from those that have not undergone previous filtering surgery.

- However, there is no study that has evaluated refractive outcomes of children with PCG that underwent cataract surgery with IOL implantation after filtering surgery.
What this study adds

- There is a greater myopic shift in eyes with PCG that undergo lens aspiration with IOL implantation and the refractive outcomes are more unpredictable compared with children operated for congenital cataract alone.

Funding No financial support has been received by any authors and none of the authors has any financial or proprietary interest in the subject matter presented.

\section{Compliance with ethical standards}

Conflict of interest The authors declare that they have no conflict of interest.

\section{References}

1. Jaffar M Care of the infantile glaucoma patient. In: Reineck RD, editor. Ophthalmol Annual. New York: Raven Press 1988. p. 15.

2. Gencik A, Gencikova A, Ferak V. Population genetical aspects of primary congenital glaucoma. I. Incidence, prevalence, gene frequency, and age of onset. Hum Genet. 1982;61:193-7.

3. Dandona L, Williams JD, Williams BC, Rao GN. Populationbased assessment of childhood blindness in southern India. Arch Ophthalmol. 1998;116:545-6.

4. Papadopoulos MKP. Childhood glaucoma. New York: Elsevier Saunders; 2005.

5. Khaw PTFS, Papadopoulos M, Wells A, Shah P. The childhood glaucomas: management. London: BMJ Books; 2000.

6. Adelman RA, Brauner SC, Afshari NA, Grosskreutz CL. Cataract formation after initial trabeculectomy in young patients. Ophthalmology. 2003;110:625-9.

7. Lichter PR, Musch DC, Gillespie BW, et al. Interim clinical outcomes in the Collaborative Initial Glaucoma Treatment Study comparing initial treatment randomized to medications or surgery. Ophthalmology. 2001;108:1943-53.

8. Tarkkanen A, Uusitalo R, Mianowicz J. Ultrasonographic biometry in congenital glaucoma. Acta Ophthalmol (Copenh). 1983;61:618-23.

9. Law SK, Bui D, Caprioli J. Serial axial length measurements in congenital glaucoma. Am J Ophthalmol. 2001;132:926-8.

10. Cashwell LF, Martin CA. Axial length decrease accompanying successful glaucoma filtration surgery. Ophthalmology. 1999;106:2307-11.

11. Kook MS, Kim HB, Lee SU. Short-term effect of mitomycin-C augmented trabeculectomy on axial length and corneal astigmatism. J Cataract Refract Surg. 2001;27:518-23.

12. Francis BA, Wang M, Lei $\mathrm{H}$, et al. Changes in axial length following trabeculectomy and glaucoma drainage device surgery. $\mathrm{Br}$ J Ophthalmol. 2005;89:17-20.

13. Gimbel HV, Ferensowicz M, Raanan M, DeLuca M. Implantation in children. J Pediatr Ophthalmol Strabismus. 1993;30:69-79.

14. Plager DA, Kipfer H, Sprunger DT, et al. Refractive change in pediatric pseudophakia: 6-year follow-up. J Cataract Refract Surg. 2002;28:810-5.

15. Crouch ER, Crouch ER Jr., Pressman SH. Prospective analysis of pediatric pseudophakia: myopic shift and postoperative outcomes. J AAPOS. 2002;6:277-82.

16. Eissa SA. Management of pseudophakic myopic anisometropic amblyopia with piggyback Visian(R) implantable collamer lens. Acta Ophthalmol. 2017;95:188-93. 
17. Enyedi LB, Peterseim MW, Freedman SF, Buckley EG. Refractive changes after pediatric intraocular lens implantation. Am J Ophthalmol. 1998;126:772-81.

18. Robb RM. Refractive errors associated with hemangiomas of the eyelids and orbit in infancy. Am J Ophthalmol. 1977;83:52-8.

19. O'Leary DJ, Millodot M. Eyelid closure causes myopia in humans. Experientia. 1979;35:1478-9.

20. Hoyt CS, Stone RD, Fromer C, Billson FA. Monocular axial myopia associated with neonatal eyelid closure in human infants. Am J Ophthalmol. 1981;91:197-200.

21. Dirani M, Tong L, Gazzard G, et al. Outdoor activity and myopia in Singapore teenage children. Br J Ophthalmol. 2009;93:997-1000.

22. Trivedi RH, Wilson ME Jr. Changes in interocular axial length after pediatric cataract surgery. J AAPOS. 2007;11:225-9.

23. Gordon RA, Donzis PB. Refractive development of the human eye. Arch Ophthalmol. 1985;103:785-9.

24. Leydolt C, Findl O, Drexler W. Effects of change in intraocular pressure on axial eye length and lens position. Eye (Lond). 2008;22:657-61.

25. McMonnies CW. An examination of the baropathic nature of axial myopia. Clin Exp Optom. 2014;97:116-24.
26. Sanders DR, Retzlaff JA, Kraff MC, et al. Comparison of the SRK/T formula and other theoretical and regression formulas. J Cataract Refract Surg. 1990;16:341-6.

27. Wang XG, Dong J, Pu YL, et al. Comparison axial length measurements from three biometric instruments in high myopia. Int $\mathrm{J}$ Ophthalmol. 2016;9:876-80.

28. Wang JK, Hu CY, Chang SW. Intraocular lens power calculation using the IOLMaster and various formulas in eyes with long axial length. J Cataract Refract Surg. 2008;34:262-7.

29. Zhang Y, Liang XY, Liu S, et al. Accuracy of intraocular lens power calculation formulas for highly myopic eyes. J Ophthalmol. 2016;2016:1917268.

30. Kohnen S, Brauweiler P. First results of cataract surgery and implantation of negative power intraocular lenses in highly myopic eyes. J Cataract Refract Surg. 1996;22:416-20.

31. Sukhija J, Kaur S, Pandav SS, et al. Pediatric cataract surgery in buphthalmos. Eur J Ophthalmol. 2015;25:260-2.

32. Gupta S, Gogia V, Jose C, et al. Peripheral retinal degenerations and rhegmatogenous detachment in primary congenital glaucoma. Retina. 2016;36:188-91. 